\title{
Factors Associated with Depression Assessed by the Patient Health Questionnaire-2 in Long-Term Cancer Survivors
}

\author{
Ae-Jin Goo, Yun-Mi Song*, Jinyoung Shin, Hyeonyoung Ko \\ Department of Family Medicine, Samsung Medical Center, Sungkyunkwan University School of Medicine, Seoul, Korea
}

\begin{abstract}
Background: This cross-sectional study aimed to evaluate the prevalence of depressive disorders and factors associated in long-term cancer survivors.

Methods: A total of 702 long-term cancer survivors over 5-years in remission were recruited in a university-affiliated tertiary hospital in Korea. Self-report using the Patient Health Questionnaire-2 and the Fatigue Severity Scale assessed depression and fatigue, respectively. Demographic characteristics, cancer-related clinical characteristics, comorbidity, health behaviors, and physical symptoms were assessed through the review of medical records or a structured self-administered questionnaire.

Results: We identified $26.1 \%$ of patients who had a depressed mood or displayed a loss of interest. The most prevalent primary site of cancer was the stomach (65.2\%), followed by lung, breast, colorectal, and thyroid cancer. We also found that $5.7 \%$ of subjects experienced double or triple primary cancers. Larger proportion among depressive group (89.1\%) complained at least one physical problem than among non-depressive group (53.2\%). Physical symptoms including sleep problems, dry mouth, indigestion, pain, decreased appetite, and febrile sense were more frequent in the depressive group than in the non-depressive group. The Fatigue Severity Scale scores were higher in the depressive group than in the non-depressive group $(\mathrm{P}<0.001)$. Multiple logistic regression analysis showed that the highest tertile level of fatigue (odds ratio, 7.31; 95\% confidence interval, 3.81-14.02) was associated with the increased risk of depression.

Conclusion: These findings suggest that careful concern about depression is necessary in long-term cancer survivors. Fatigue may be a surrogate sign for depression, and warrants further evaluation.
\end{abstract}

Keywords: Survivors; Depression; Fatigue; Koreans 


\section{INTRODUCTION}

Cancer is a critical and severe disease. However, the survival rate of cancer patients is increasing world-wide as diagnosis and treatment technology improve. ${ }^{1)}$ According to the National Cancer Registration, approximately 1 million people who were diagnosed with cancer between 1993 and 2011 survived past December 31st 2012. Furthermore, there were more than 40,000 long-term cancer survivors 5 years postdiagnosis. ${ }^{2)}$

Long-term cancer survivors also often have other chronic diseases as well as deterioration of physical function. ${ }^{3)}$ It is reported that psychosocial problems are common as well. ${ }^{3)}$ Depression is a very important psychosocial health problem that lowers the quality of life, and increases the desire for hastened death and death rate in both cancer patients and long-term cancer survivors. ${ }^{4)}$ However, depression in cancer survivors may be underestimated because depression may appear different from the normal population. This research assesses the level of depression using the Patient Health Questionnaire-2 (PHQ$2)^{5)}$ and we evaluate factors associated with depression in long-term cancer survivors.

\section{METHODS}

\section{Participants and Research Variables}

This is a cross-sectional study on adults (>19 year-old) long-term cancer survivors living for 5 years after their final treatment. Cancer survivors were recruited from a cancer survivor clinic of the university-affiliated tertiary hospital in Korea between July 2013 and September 2014. Data were collected through the review of medical records or a structured self-administered questionnaire. Of a total of 890 cancer survivors, 702 were included in the final analysis, and 184 people for whom 5 years since their last treatment had not yet passed, were excluded. Four additional people were also excluded because of lack of data.

Collected variables were age, gender, medical history (e.g., diagnosis of cancer and history of cancer treatment), family history of cancer, physical symptoms, depressive symptoms, Fatigue Severity Scale, alcohol intake, smoking status, physical exercise, comorbidity (e.g., hypertension, diabetes mellitus, dyslipidemia, osteoporosis, cardiovascular disease, liver disease, and stroke), previous influenza vaccination, and body measures (e.g., blood pressure, weight, and height).

Depression was evaluated by using PHQ-2 (yes/no type) that consisted of two questions: "I am in agony because I feel low, depressed and hopeless" and "I am in agony because I do not feel happy and have lost interest in everything." ${ }^{\text {6) }}$ PHQ-2 is clinically convenient to use because of its short time consumption. There are two types of scales (short-answer type and score type) depending on evaluation method. ${ }^{6}$ ) The sensitivity and specificity of the short-answer type PHQ-2 in screening depression is reported to be $96 \%$ and $57 \%$, respectively. ${ }^{7)}$ Subjects who answered "yes" to at least one of the questions were categorized as "depressive group (PHQ-2 score $\geq 1$ )," and subjects who answered "no" to both questions were categorized as "non-depressive group (PHQ-2 score=0)."

Fatigue severity was evaluated using the Fatigue Severity Scale (FSS). ${ }^{8)}$ FSS consists of 9 questions on various fatigue symptoms experienced since the last visit. Patients chose on a scale between "strongly disagree (1 point)" and "strongly agree (7 point)." Each item on the questionnaire was then added together. Subjects were split into 3 groups (low [9-17], intermediate [18-30], and high [31-63]) according to FSS score distribution (9-63 points).

Weight and height were measured using a GL-310P scale (G-Tech International Co., Uijeongbu, Korea). Body mass index (BMI, $\mathrm{kg} / \mathrm{m}^{2}$ ) was calculated by dividing the measured weight $(\mathrm{kg})$ by square of height $\left(\mathrm{m}^{2}\right)$, and patients were placed into 3 groups: (1) low weight group (BMI <18.5), (2) normal weight group (BMI 18.5-22.9), and (3) overweight/obesity group (BMI $\geq 23.0$ ).

A family history of cancer, health behavior, previous influenza vaccination, comorbidity, and physical symptoms were surveyed using the structured self-administered questionnaire. Physical symptoms were investigated through corresponding symptoms. Symptoms were assessed by asking the following questions: "I have no appetite," "I lose weight without special reason," "My mouth is very dry," "I have a fever or chill," "I am out of breath after a small activity," "I have trouble with my digestion," "My excrement became thin or my bowel habits changed," "I have swollen, painful joints," "I have a headache," "I have a sexual problem," "I have a tightening feeling, pressure, or pain in my chest," and "I have a hard time sleeping and I wake up easily." Patients were also categorized by smoking habits: non-smoker, former-smoker, or current-smoker, and alcohol use: non-drinker, former-drinker, or current-drinker. Patients were also categorized into a non-exercise group (group without any exercise), inadequate-exercise group (average total exercise time per week $<150$ minutes), or adequate-exercise group (average total exercise time per week $\geq 150$ minutes).

This research is performed with the approval of the institutional review board of Samsung Medical Center (SMC2014-12-106).

\section{Statistical Analysis}

Demographic and clinical characteristic between non-depressive and depressive groups were compared using a two-tailed t-test and a chisquare test. Independent factors related to depression were evaluated using multiple logistic regression analysis. The regression model includes variables such as sex, age, BMI, drinking, smoking, exercise, comorbidity, FSS score, and cancer recurrence experience. These factors were selected as a result of univariate analysis, and the correspondence among health condition, prognosis, and quality of life was found in the existing research. ${ }^{9)}$

All analyses were done using PASW SPSS ver. 18.0 (SPSS Inc., Chicago, IL, USA). Significance level was set to $\mathrm{P}<0.05$ and significance level for the multiple logistic regression was set to $\mathrm{P}<0.00555$ in consideration for the Bonferroni correction. 


\section{RESULTS}

Among the participants, the number of non-depressive group and depressive group were 519 people (73.9\%) and 183 people (26.1\%), respectively. Average age \pm standard deviation of those two groups was $62.0 \pm 10.4$ and $60.5 \pm 11.4$ years old. There was no significant difference in age groups. The proportion of females in the depressive group was $60.1 \%$, and was significantly higher than the proportion of females in the non-depressive group $(38.0 \%, \mathrm{P}<0.001)$.

\section{General Characteristics of Participants}

In male participants, there were no significant differences between depressive and non-depressive in average age, BMI, previous influenza vaccination, drinking habits, and exercise habits. There was a significant difference in smoking habits $(\mathrm{P}<0.001)$ : more never-smoked and current-smokers were in the depressive group, and more formersmokers were found in the non-depressive group $(\mathrm{P}=0.014)$. Hypertension, diabetes mellitus, and dyslipidemia were commonly observed as comorbid illness in men. The prevalence of hypertension $(\mathrm{P}=0.040)$ and dyslipidemia $(\mathrm{P}<0.001)$ was significantly higher in the depressive group compared to the non-depressive group in men (Table 1).

There were no significant differences in average age, distribution of body mass index, previous influenza vaccination, smoking habits, exercise habits between females in the depressive and females in the non-depressive group. However, there was a significant difference in drinking habits $(\mathrm{P}=0.016)$. The majority of female former-drinkers and current-drinkers were found in the depressive group, whereas, many never-drinkers were found in the non-depressive group. Hypertension, dyslipidemia, osteoporosis, and diabetes mellitus were common comorbid illnesses in female patients. However, there was no significant difference in the proportion of survivors with more than one coexisting disease between the depressive group (45.5\%) and the nondepressive group (45.7\%). However, diabetes mellitus was more common in the depressive group referring to the distribution of individual diseases $(\mathrm{P}=0.024)$.

\section{Cancer-Related Clinical Characteristics}

The majority of participants (94.3\%) have experienced primary cancer

Table 1. Baseline characteristics of study participants by the presence of depression*

\begin{tabular}{|c|c|c|c|c|}
\hline \multirow{2}{*}{ Characteristic } & \multicolumn{2}{|c|}{ Men } & \multicolumn{2}{|c|}{ Women } \\
\hline & Non-depressive group ( $\mathrm{n}=322$ ) & Depressive group $(\mathrm{n}=73$ ) & Non-depressive group ( $\mathrm{n}=197$ ) & Depressive group $(\mathrm{n}=110)$ \\
\hline Age (y) & $63.5 \pm 9.5$ & $61.3 \pm 11.3$ & $59.4 \pm 11.3$ & $60.0 \pm 11.4$ \\
\hline \multicolumn{5}{|l|}{ Body mass index ${ }^{\dagger}\left(\mathrm{kg} / \mathrm{m}^{2}\right)$} \\
\hline$<18.5$ & $18(5.8)$ & $4(5.8)$ & $22(11.6)$ & $16(14.8)$ \\
\hline $18.5-22.9$ & $176(56.6)$ & $37(53.6)$ & $110(58.2)$ & $61(56.5)$ \\
\hline$\geq 23.0$ & $117(37.6)$ & $28(40.6)$ & 57 (30.2) & $31(28.7)$ \\
\hline \multicolumn{5}{|c|}{ Previous influenza vaccination ${ }^{\dagger}$} \\
\hline No & $124(38.5)$ & $34(46.6)$ & $68(34.7)$ & $43(39.4)$ \\
\hline Yes & $198(61.5)$ & $39(53.4)$ & $128(65.3)$ & $66(60.6)$ \\
\hline \multicolumn{5}{|l|}{ Smoking status ${ }^{\dagger}$} \\
\hline None & 29 (10.4) & $19(26.8)^{\ddagger}$ & $161(93.6)$ & 87 (93.5) \\
\hline Former & $228(81.4)$ & $42(59.2)^{\ddagger}$ & $10(5.8)$ & $4(4.3)$ \\
\hline Current & $23(8.2)$ & $10(14.1)^{\ddagger}$ & $1(0.6)$ & $2(2.2)$ \\
\hline \multicolumn{5}{|l|}{ Alcohol intake $^{\dagger}$} \\
\hline None & $18(6.5)$ & 7 (10.4) & $80(46.0)$ & $24(27.6)^{\ddagger}$ \\
\hline Former & $136(48.9)$ & $34(50.7)$ & $63(36.2)$ & $44(50.6)^{\ddagger}$ \\
\hline Current & $124(44.6)$ & $26(38.8)$ & $31(17.8)$ & $19(21.8)^{\ddagger}$ \\
\hline \multicolumn{5}{|l|}{ Physical exercise ${ }^{\dagger, \S}$} \\
\hline No & $32(9.9)$ & $4(5.5)$ & $35(17.8)$ & $24(21.8)$ \\
\hline Yes (inadequate) & $87(27.0)$ & $26(35.6)$ & $47(23.9)$ & 31 (28.2) \\
\hline Yes (adequate) $)^{\S}$ & $203(63.0)$ & $43(58.9)$ & $115(58.4)$ & $55(50.0)$ \\
\hline \multicolumn{5}{|l|}{ Comorbidity" } \\
\hline Hypertension & $51(15.8)$ & $19(26.0)^{\ddagger}$ & 39 (19.8) & $14(12.7)$ \\
\hline Diabetes mellitus & $40(12.4)$ & $8(11.0)$ & $17(8.6)$ & $19(17.3)^{\ddagger}$ \\
\hline Dyslipidemia & $23(7.1)$ & $15(20.5)^{\ddagger}$ & $28(14.2)$ & $12(10.9)$ \\
\hline Osteoporosis & $5(1.6)$ & $3(4.1)$ & $19(9.6)$ & $17(15.5)$ \\
\hline Cardiovascular disease & $19(5.9)$ & $4(5.5)$ & $2(1.0)$ & $1(0.9)$ \\
\hline Liver disease & $6(1.9)$ & $1(1.4)$ & $3(1.5)$ & $4(3.6)$ \\
\hline Stroke & $3(0.9)$ & $2(2.7)$ & $3(1.5)$ & $1(0.9)$ \\
\hline
\end{tabular}

Values are presented as mean \pm standard deviation or number (\%).

PHQ, Patient Health Questionnaire.

*Defined by PHQ-2. The depressive group had a PHQ-2 score of 1 or 2, and the non-depressive group had a PHQ-2 score of 0 . 'For some participants, information was not available. ${ }^{\mathrm{P}}$-value for difference between depressive and non-depressive group $<0.05$, obtained by t-test for continuous variables and chi-square test for categorical variables. ${ }^{\S}$ Doing physical exercise for $150 \mathrm{~min} / \mathrm{wk}$ or longer. "Participants were able to choose multiple responses. 
Table 2. Distribution of cancer-related clinical characteristics by the presence of depressiveness*

\begin{tabular}{|c|c|c|c|}
\hline Variable & $\begin{array}{l}\text { Non-depressive } \\
\text { group }(n=519)\end{array}$ & $\begin{array}{c}\text { Depressive } \\
\text { group }(n=183)\end{array}$ & P-value ${ }^{\dagger}$ \\
\hline No. of primary cancer site & & & 0.340 \\
\hline 1 & $492(94.8)$ & $170(92.9)$ & \\
\hline$\geq 2$ & 27 (5.2) & $13(7.1)$ & \\
\hline Location of cancer & & & 0.385 \\
\hline Stomach & 328 (63.2) & $102(55.7)$ & \\
\hline Lung & $83(16.0)$ & $21(11.5)$ & \\
\hline Breast & $29(5.6)$ & $11(6.0)$ & \\
\hline Colorectal & $26(5.0)$ & $14(7.7)$ & \\
\hline Thyroid & $19(3.7)$ & $8(4.4)$ & \\
\hline Other ${ }^{\ddagger}$ & $34(6.6)$ & $27(14.8)$ & \\
\hline Stage of cancer ${ }^{\S}$ ॥l & & & 0.384 \\
\hline $0-1$ & $317(61.1)$ & $106(57.9)$ & \\
\hline$\|$ & $89(17.1)$ & $33(18.0)$ & \\
\hline III-IV & $74(14.3)$ & $23(12.6)$ & \\
\hline Unknown & $39(7.5)$ & $21(11.5)$ & \\
\hline Family history of cancer & & & 0.175 \\
\hline No & $387(74.6)$ & $127(69.4)$ & \\
\hline Yes & $132(25.4)$ & $56(30.6)$ & \\
\hline Cancer recurrence $^{\S}$ & & & 0.043 \\
\hline No & 507 (98.3) & $172(95.6)$ & \\
\hline Yes & $9(1.7)$ & $8(4.4)$ & \\
\hline \multicolumn{4}{|l|}{ Type of treatment ${ }^{\S}$} \\
\hline Surgery & $510(98.6)$ & $179(97.8)$ & 0.437 \\
\hline Chemotherapy & $187(36.2)$ & $64(35.4)$ & 0.845 \\
\hline Radiotherapy & $132(25.5)$ & $42(23.2)$ & 0.533 \\
\hline Time since treatment ${ }^{\S}(y)$ & $8.1 \pm 3.2$ & $8.0 \pm 2.2$ & 0.570 \\
\hline
\end{tabular}

Values are presented as mean \pm standard deviation or number (\%).

PHQ, Patient Health Questionnaire.

*Defined by PHQ-2. The depressive group had a PHQ-2 score of 1 or 2, and the nondepressive group had a PHQ-2 score of $0 .{ }^{\dagger}$ Obtained by $\mathrm{t}$-test for continuous variables and chi-square test for categorical variables. ${ }^{\ddagger} 0$ ther cancer includes esophageal $(n=19)$, head and neck $(n=9)$, hepatobiliary $(n=9)$, genitourinary $(n=10)$, gynecologic $(n=5)$, lymphoma $(n=5)$, soft tissue sarcoma $(n=2)$, skin $(n=1)$, and ophthalmic $(n=1)$. Except these cases, chi-square test was performed. ${ }^{\circledR}$ For some participants, information was not available. "If a patient has cancer at two or more sites with different stages, the highest stage was selected.

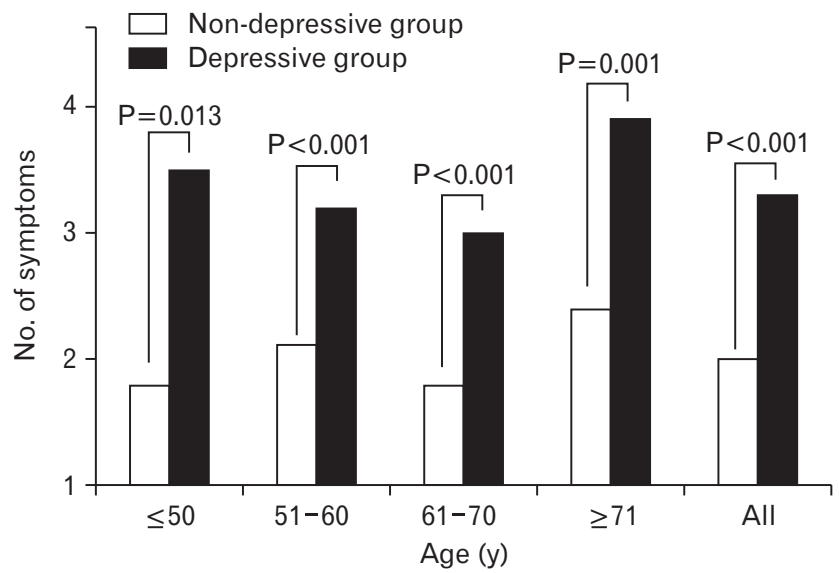

Figure 1. Comparisons of the number of physical symptoms between the nondepressive and the depressive group. Depression was defined by the PHQ-2. The depressive group had a PHQ-2 score of 1 or 2, and the non-depressive group had a PHQ-2 score of 0 . P-value for the differences between non-depressive and depressive group was obtained by t-test. PHQ, Patient Health Questionnaire. in one organ of the body. There was no significant difference of primary cancer number between non-depressive and depressive groups. Stomach cancer was the largest form of cancer in patients followed by lung, breast, colorectal and thyroid. There was no significant difference in site of cancer between the two groups. Furthermore, no noticeable differences between the two groups were found on stage of cancer, family history of cancer, experienced therapy method, or elapsed time after discontinuation of treatments. Experience of cancer recurrence was significantly higher in the depressive group compared to the nondepressive group $(\mathrm{P}=0.043)$ (Table 2).

Table 3. Patterns of physical symptoms by the presence of depressiveness ${ }^{*,}$

\begin{tabular}{lccr}
\hline \multicolumn{1}{c}{ Physical symptoms } & $\begin{array}{c}\text { Non-depressive } \\
\text { group }(\mathrm{n}=519)\end{array}$ & $\begin{array}{c}\text { Depressive } \\
\text { group }(\mathrm{n}=183)\end{array}$ & P-value $^{\ddagger}$ \\
\hline No specific symptoms & $243(46.8)$ & $20(10.9)$ & $<0.001$ \\
Sleep problem & $96(34.8)$ & $86(52.8)$ & $<0.001$ \\
Respiratory problem & $67(24.3)$ & $49(30.1)$ & 0.184 \\
Dry mouth & $59(21.4)$ & $56(34.4)$ & 0.003 \\
Indigestion & $55(19.9)$ & $60(36.8)$ & $<0.001$ \\
Headache & $47(17.0)$ & $64(39.3)$ & $<0.001$ \\
Bowel habit change & $52(18.8)$ & $38(23.3)$ & 0.262 \\
Chest pain & $43(15.6)$ & $42(25.8)$ & 0.009 \\
Sexual dysfunction & $39(14.1)$ & $25(15.3)$ & 0.729 \\
Arthralgia & $36(13.0)$ & $34(20.9)$ & 0.031 \\
Weight loss & $31(11.2)$ & $23(14.1)$ & 0.375 \\
Decreased appetite & $19(6.9)$ & $35(21.5)$ & $<0.001$ \\
Febrile seizure & $17(6.2)$ & $27(16.6)$ & $<0.001$ \\
\hline
\end{tabular}

Values are presented as number (\%).

$P H Q$, Patient Health Questionnaire.

*Defined by PHQ-2. The depressive group had a PHQ-2 score of 1 or 2, and the nondepressive group had a PHQ-2 score of $0 .{ }^{\dagger}$ Some participants had more than one physical symptom. ${ }^{\ddagger}$ Obtained by chi-square test.

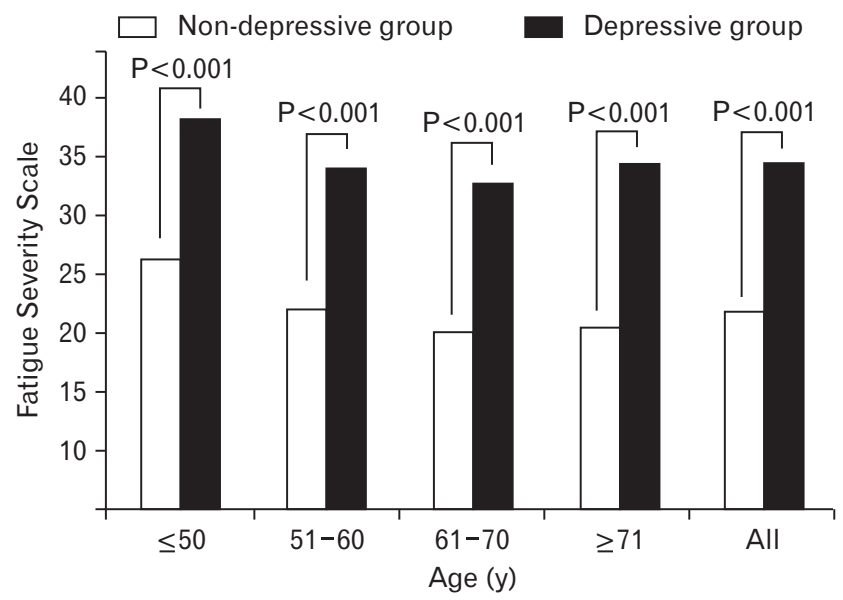

Figure 2. Comparisons of Fatigue Severity Scale score between non-depressive and depressive group. Depression was defined by PHQ-2. The depressive group had a PHQ-2 score of 1 or 2, and the non-depressive group had a PHQ-2 score of 0 . Fatigue Severity Scale ranges between 9 (no fatigue) and 63 (most fatigue). The $P$-value for the differences between non-depressive and depressive groups was obtained by t-test. PHQ, Patient Health Questionnaire. 


\section{Distinctions of Physical Symptoms by the Presence of Depressive Symptoms}

The presence of more than one physical symptom in the depressive group (89.1\%) was significantly higher compared to the non-depressive group $(53.2 \%)(\mathrm{P}<0.001)$. Furthermore, the number of physical symptoms (average \pm standard deviation) was significantly higher in the depressive group (3.3 \pm 2.1$)$ than the non-depressive group (2.0 \pm 1.4 , $\mathrm{P}<0.001)$. Sleep problems, dry mouth, indigestion, pain, decreased appetite, and febrile seizure were more common in the depressive group compared to the non-depressive group. There were no significant differences between the two groups in respiratory problem, bowel habit change, sexual dysfunction, and weight loss. FSS score (average \pm standard deviation) was $34.8 \pm 14.7$ in the depressive group, and was significantly higher compared to the non-depressive group $(22.0 \pm 11.9)(\mathrm{P}<0.001)$. The same tendency was observed across age groups (Table 3 and Figures 1, 2).

\section{Factors Associated with Depressive Disorders in Long- Term Cancer Survivors}

The following are factors related to depressive disorder: female (odds ratio, 2.46; 95\% confidence interval, 1.74-3.48), intermediate fatigue severity (odds ratio, 1.82; 95\% confidence interval, 1.05-3.17), high Fatigue Severity Scale score (odds ratio, 7.32; 95\% confidence interval, $4.40-12.20$ ), and former-smoking (odds ratio, 0.35 ; $95 \%$ confidence interval, 0.23-0.51) (Table 4).
The influence of other variables was found using multiple logistic regression and evaluating independent associations. Inadequate exercise (odds ratio, 2.16; 95\% confidence interval, 1.03-4.55) and high FSS score (odds ratio, 7.31; 95\% confidence interval, 3.81-14.02) had a significant association with depression. However, a high FSS score was the only variable associated with depression when the level of significance was $\mathrm{P}<0.00555$ following a Bonferroni correction. No significant association was found between depressive symptoms and other variables including age, degree of obesity, smoking status, drinking status, comorbidity of illness, and experience of cancer recurrence.

\section{DISCUSSION}

Depression is a common symptom experienced by cancer patients. ${ }^{10)}$ However, it is unknown if the prevalence of depression in long-term cancer survivors is significantly higher than in general population. According to a systematic review by Walker et al., ${ }^{11)}$ the prevalence of depression in patients in palliative care was $7 \%-49 \%$. However, the prevalence of depression in cancer survivors was $5 \%-16 \%$ for outpatients, and $4 \%-14 \%$ for inpatients. This indicates that the prevalence of depression was not significantly higher in cancer patients than in general population. In a long-term follow-up study performed by Burgess et al., ${ }^{12)}$ annual prevalence of depression during the first year after cancer diagnosis was $48 \%$, which is significantly higher than the depression prevalence in non-cancer population. However, the prevalence of de-

Table 4. Factors associated with depressiveness ${ }^{*}$ of cancer survivors

\begin{tabular}{|c|c|c|c|c|c|}
\hline \multirow{2}{*}{ Variable } & \multirow{2}{*}{ Category } & \multicolumn{2}{|c|}{ Unadjusted } & \multicolumn{2}{|c|}{ Multi-variable adjusted } \\
\hline & & OR $(95 \% \mathrm{Cl})^{\dagger}$ & P-value & $\mathrm{OR}(95 \% \mathrm{Cl})^{\dagger}$ & P-value \\
\hline Sex & Women & $2.46(1.74-3.48)$ & $<0.001$ & $1.19(0.53-2.64)$ & 0.674 \\
\hline Age, 1-year & & $0.99(0.97-1.00)$ & 0.122 & $1.00(0.97-1.02)$ & 0.751 \\
\hline \multirow[t]{3}{*}{ Body mass index $\left(\mathrm{kg} / \mathrm{m}^{2}\right)$} & $<18.5$ & $1.46(0.81-2.62)$ & 0.205 & $0.97(0.44-2.17)$ & 0.943 \\
\hline & $18.5-22.9$ & 1.00 & & 1.00 & \\
\hline & $\geq 23.0$ & $0.99(0.68-1.44)$ & 0.956 & $1.40(0.84-2.32)$ & 0.199 \\
\hline \multirow[t]{3}{*}{ Smoking } & Never & 1.00 & & 1.00 & \\
\hline & Former & $0.35(0.23-0.51)$ & $<0.001$ & $0.59(0.26-1.32)$ & 0.198 \\
\hline & Current & $0.90(0.43-1.87)$ & 0.769 & $1.65(0.54-5.08)$ & 0.381 \\
\hline \multirow[t]{3}{*}{ Alcohol intake } & Never & 1.00 & & 1.00 & \\
\hline & Former & $1.24(0.77-2.01)$ & 0.383 & $1.25(0.66-2.39)$ & 0.497 \\
\hline & Current & $0.92(0.54-1.55)$ & 0.748 & $1.15(0.55-2.41)$ & 0.720 \\
\hline \multirow[t]{3}{*}{ Exercise } & No & 1.00 & & 1.00 & \\
\hline & Inadequate & $1.02(0.59-1.75)$ & 0.949 & $2.16(1.03-4.55)$ & 0.043 \\
\hline & Adequate $^{\ddagger}$ & $0.74(0.45-1.21)$ & 0.229 & $1.20(0.60-2.37)$ & 0.607 \\
\hline \multirow[t]{2}{*}{ Comorbidity } & No & 1.00 & & 1.00 & \\
\hline & Yes & $1.36(0.97-1.90)$ & 0.077 & $1.22(0.75-2.00)$ & 0.426 \\
\hline \multirow[t]{2}{*}{ Cancer recurrence } & No & 1.00 & & 1.00 & \\
\hline & Yes & $2.62(1.00-6.88)$ & 0.052 & $3.35(0.72-15.57)$ & 0.122 \\
\hline \multirow[t]{3}{*}{$\mathrm{FSS}^{\S}$} & Low & 1.00 & & 1.00 & \\
\hline & Intermediate & $1.82(1.05-3.17)$ & 0.033 & $1.73(0.88-3.41)$ & 0.112 \\
\hline & High & $7.32(4.40-12.20)$ & $<0.001$ & $7.31(3.81-14.02)$ & $<0.001$ \\
\hline
\end{tabular}

OR, odds ratio; Cl, confidence interval; PHQ, Patient Health Questionnaire; FSS, Fatigue Severity Scale.

*Defined by PHQ-2. The depressive group had a PHQ-2 score of 1 or 2 , and the non-depressive group had a PHQ-2 score of 0 . 'Estimated by multiple logistic regression analysis. ${ }^{\star}$ Doing physical exercise for 150 min/wk or longer. §FSS ranges between 9 (no fatigue) and 63 (most fatigue). Categorized into three groups by the tertile distribution such as low (9-17, $n=198)$, intermediate (18-30, $n=194)$, and high $(31-63, n=197)$. 
pression decreased annually and was $15 \%$ on last fifth year, which is not significantly different compared to other people. A meta-analysis by Mitchell et al., ${ }^{13)}$ revealed that the prevalence of depression in cancer survivors was $11.6 \%$ (95\% confidence interval, 7.7\%-16.2\%). Khan et al. ${ }^{14)}$ compared antidepressant treatment and counseling between ordinary people and breast, colorectal and prostate cancer survivors living for 5 years since diagnosis. He found no significant differences between the two groups.

In the present study, we observed that $26.1 \%$ of long-term cancer survivors have depressive symptoms. Kim et al. ${ }^{9)}$ evaluated breast cancer survivors $(n=1,933)$ with an average of 4.6 years since initial diagnosis using Beck Depression Inventory (BDI). They found that $24.9 \%$ of cancer survivors had severe depression (BDI $\geq 19$ ). In another study from a hospital focusing on cervical cancer survivors ( $\mathrm{n}=828$ ) living an average of 6.9 years after diagnosis, Kim et al. ${ }^{15)}$ used the Hospital Anxiety and Depression Scale-D (HADS-D), and found that $34.6 \%$ of participants had depression (HADS-D $\geq 8$ ). The current study evaluated depressive symptoms using PHQ-2, whereas other studies evaluate with BDI or HADS-D. Thus, it is difficult to directly compare the findings between studies because of the use of different research instruments. This study uses PHQ-2 as a comparatively simpler screening tool. Therefore, the Diagnostic and Statistical Manual of Mental Disorders 5th Edition based research on depression in Korean long-term cancer survivors, and validity evaluation of PHQ-2 on cancer survivors are needed.

Moreover, these studies were performed in a hospital, and there is a high possibility of over-evaluation in the severity of the symptoms in these patients compared to patients who did not visit the hospital. Therefore, additional research using more accurate evaluation tools based on the local society is needed for more precise evaluation of depression in Korean long-term cancer survivors.

In research by Zabora et al. ${ }^{16)}$ based on recently-cancer-diagnosed people and long-term survivors ( $14 \%$ of them survived 4 years or more after diagnosis and remaining $86 \%$ survived less than 4 years), the score of Brief Symptom Inventory-depression subscales were high in pancreatic cancer, lung cancer, liver cancer, hematologic malignancy survivors, and low in skin cancer, prostate cancer, gynecologic cancer, breast cancer survivor. Distress including anxiety and depression had high prevalence rates in survivors from lung cancer (43.4\%), brain cancer (42.7\%), Hodgkin's lymphoma (37.8\%), and pancreatic cancer (36.6\%). A systematic review by Massie et al. ${ }^{17)}$ reported that the frequency of depression was high in survivors from oral cavity, pharynx, and larynx cancer (22\%-57\%), pancreatic cancer (33\%-50\%), breast cancer $(1.5 \%-46 \%)$ and lung cancer (11\%-44\%). According to these results, depression symptoms can co-occur with cancer leading to a lower quality of life. However, the period of time following cancer treatment included in our study was on average 8 years, which is longer survival time compared to other patients. No pancreatic cancer survivor (0 patients) was included, and a relatively low number of head and neck cancer survivors (9 patients) were included. Thus, there are difficulties in evaluating these factors.
We assume that cancer recurrence has a high possibility of causing depressive symptoms because of fear of additional recurrent cancer, or fear of serious consequences. However, previous meta-analysis reports have shown no significant relationships between cancer recurrence and depression. ${ }^{18)}$ Univariate analysis from this study showed a significant relationship between depression and cancer recurrence among characteristics associated with cancer. However, the relationship was not significant following the corrections of other factors.

In this study, we have observed that depression is particularly related to sleep problems, dry mouth, indigestion, headache, chest pain, arthralgia, decreased appetite, and febrile seizure. Kim et al. ${ }^{9)}$ observed that decreased appetite (odds ratio, 3.68; 95\% confidence interval, 1.89-7.16), sleep problem (odds ratio, 2.94; 95\% confidence interval, 2.04-4.24), constipation (odds ratio, 2.37; 95\% confidence interval, 1.58-3.57), and respiratory problems (odds ratio, 1.63; 95\% confidence interval, 1.01-2.64) had a high relationship with depression. No significant relationship was found between somatic pain and depression. Both our study and Kim et al. ${ }^{9}$ used a cross-sectional design. Thus, the relationship on temporal sequence of occurrence could not be identified. Therefore, it is difficult to identify whether physical symptoms cause depression, or if a depressed psychological state causes more physical symptoms. However, findings from existing studies including ours imply that physical symptoms can be used to recognize depression in cancer survivors.

Fatigue is a common health problem among cancer patients in recovery and cancer survivors. ${ }^{19,20)}$ A significant relationship was found between fatigue and depression after the consideration of other factors. Fatigue is one of the diagnostic components of depression. However, fatigue is recognized as a physical symptom in both patients and clinicians. Thus, diagnosis of depression is often overlooked. ${ }^{21)}$ The findings from our research suggest the importance of active evaluation regarding depression with patients who feel fatigued.

Previous research found that long-term cancer survivors living for more than 5 years had a decline in exercise ability (odds ratio, 1.49; 95\% confidence interval, 1.03-2.08) and they had difficulty in beginning exercise (odds ratio, 1.43; 95\% confidence interval, 1.11-1.85). ${ }^{22}$ From the research by Smith et al., ${ }^{23)}$ the case of long-term cancer survivors who did not follow the adequate level of physical exercise recommended by Centers for Disease Control and Prevention was 1.7 time higher than the larger population. Cancer survivors are affected by the adverse effect by cancer treatment, fatigue, pains, depression and anxiety. ${ }^{24)}$ However, more than half of patients from the depressive and non-depressive groups of our study exercised for 150 minutes or more a week. This is not lower than the exercise participation rate of people observed in the Korea National Health and Nutrition Examination Survey. ${ }^{25)}$

Smoking rate of males and females observed in our study was lower than the smoking rate of people studied in the Korea National Health and Nutrition Examination Survey. It is also lower than the smoking rate of cancer survivors observed in the Korea National Health and Nutrition Examination Survey. ${ }^{26)}$ This difference may have occurred 
because our research was performed on hospital visitors including people with more desirable health behavior.

This study has a few limitations. First, it is a cross-sectional study, and it is hard to identify the relationship on temporal sequence between studied factors and depression. Second, patients were recruited from a hospital, and not from community. There is the possibility we overestimated depressive symptoms or physical symptoms, and health behavior overall can be biased. Third, depression was evaluated by a relatively simple screening tool (PHQ-2), and may also cause overestimation of the depression. However, PHQ-2 score tends to increase as severity of depression increases. ${ }^{6}$ Furthermore, PHQ-2 as a screening tool is a test with high reliability and validity. ${ }^{7}$ Thus, we believe PHQ-2 caused no serious bias to the result of this research.

In conclusion, we observed that a considerable number of longterm cancer survivors experience depressive symptoms, and fatigue is highly related to depression. This implies that depression screenings should be done during treatment, especially to cancer survivors who have fatigue.

\section{CONFLICT OF INTEREST}

No potential conflict of interest relevant to this article was reported.

\section{ACKNOWLEDGMENTS}

This research was supported by Basic Science Research Program through the National Research Foundation of Korea (NRF) funded by the Ministry of Science, ICT and future Planning (2014R1A2A2A01002705).

\section{REFERENCES}

1. Siegel R, Ma J, Zou Z, Jemal A. Cancer statistics, 2014. CA Cancer J Clin 2014;64:9-29.

2. Jung KW, Won YJ, Kong HJ, Oh CM, Lee DH, Lee JS. Cancer statistics in Korea: incidence, mortality, survival, and prevalence in 2011. Cancer Res Treat 2014;46:109-23.

3. Stein KD, Syrjala KL, Andrykowski MA. Physical and psychological long-term and late effects of cancer. Cancer 2008;112(11 Suppl):2577-92.

4. Chochinov HM. Depression in cancer patients. Lancet Oncol 2001;2: 499-505.

5. Kroenke K, Spitzer RL, Williams JB. The Patient Health Questionnaire-2: validity of a two-item depression screener. Med Care 2003;41: 1284-92.

6. Shin JH, Kim HC, Jung CH, Kim JB, Jung SW, Cho HJ, et al. The standardization of the Korean version of the Patient Health Questionnaire-2. J Korean Neuropsychiatr Assoc 2013;52:115-21.

7. Whooley MA, Avins AL, Miranda J, Browner WS. Case-finding instruments for depression: two questions are as good as many. J Gen Intern Med 1997;12:439-45.

8. Chung KI, Song CH. Clinical usefulness of fatigue severity scale for patients with fatigue, and anxiety or depression. Korean J Psychosom Med 2001;9:164-73.
9. Kim SH, Son BH, Hwang SY, Han W, Yang JH, Lee S, et al. Fatigue and depression in disease-free breast cancer survivors: prevalence, correlates, and association with quality of life. J Pain Symptom Manage 2008;35:644-55.

10. Derogatis LR, Morrow GR, Fetting J, Penman D, Piasetsky S, Schmale $\mathrm{AM}$, et al. The prevalence of psychiatric disorders among cancer patients. JAMA 1983;249:751-7.

11. Walker J, Holm Hansen C, Martin P, Sawhney A, Thekkumpurath P, Beale C, et al. Prevalence of depression in adults with cancer: a systematic review. Ann Oncol 2013;24:895-900.

12. Burgess C, Cornelius V, Love S, Graham J, Richards M, Ramirez A. Depression and anxiety in women with early breast cancer: five year observational cohort study. BMJ 2005;330:702.

13. Mitchell AJ, Ferguson DW, Gill J, Paul J, Symonds P. Depression and anxiety in long-term cancer survivors compared with spouses and healthy controls: a systematic review and meta-analysis. Lancet Oncol 2013;14:721-32.

14. Khan NF, Ward AM, Watson E, Rose PW. Consulting and prescribing behaviour for anxiety and depression in long-term survivors of cancer in the UK. Eur J Cancer 2010;46:3339-44.

15. Kim SH, Kang S, Kim YM, Kim BG, Seong SJ, Cha SD, et al. Prevalence and predictors of anxiety and depression among cervical cancer survivors in Korea. Int J Gynecol Cancer 2010;20:1017-24.

16. Zabora J, BrintzenhofeSzoc K, Curbow B, Hooker C, Piantadosi S. The prevalence of psychological distress by cancer site. Psychooncology 2001;10:19-28.

17. Massie MJ. Prevalence of depression in patients with cancer. J Natl Cancer Inst Monogr 2004;(32):57-71.

18. Satin JR, Linden W, Phillips MJ. Depression as a predictor of disease progression and mortality in cancer patients: a meta-analysis. Cancer 2009;115:5349-61.

19. Hofman M, Ryan JL, Figueroa-Moseley CD, Jean-Pierre P, Morrow GR. Cancer-related fatigue: the scale of the problem. Oncologist 2007;12 Suppl 1:4-10.

20. Fobair P, Hoppe RT, Bloom J, Cox R, Varghese A, Spiegel D. Psychosocial problems among survivors of Hodgkin's disease. J Clin Oncol 1986;4:805-14.

21. Ford S, Fallowfield L, Lewis S. Can oncologists detect distress in their out-patients and how satisfied are they with their performance during bad news consultations? Br J Cancer 1994;70:767-70.

22. Ness KK, Wall MM, Oakes JM, Robison LL, Gurney JG. Physical performance limitations and participation restrictions among cancer survivors: a population-based study. Ann Epidemiol 2006;16:197-205.

23. Smith WA, Nolan VG, Robison LL, Hudson MM, Ness KK. Physical activity among cancer survivors and those with no history of cancer- a report from the National Health and Nutrition Examination Survey 2003-2006. Am J Transl Res 2011;3:342-50.

24. Maunsell E, Pogany L, Barrera M, Shaw AK, Speechley KN. Quality of life among long-term adolescent and adult survivors of childhood cancer. J Clin Oncol 2006;24:2527-35.

25. Kang K, Sung J, Kim CY. High risk groups in health behavior defined by clustering of smoking, alcohol, and exercise habits: National Heath and Nutrition Examination Survey. J Prev Med Public Health 2010;43:73-83.

26. Kim H, Kim MH, Park YS, Shin JY, Song YM. Factors that predict persistent smoking of cancer survivors. J Korean Med Sci 2015;30:853-9. 